\title{
Image Analysis of Nailfold Capillary Patterns from Video Sequences
}

\author{
P.D. Allen ${ }^{1}$, C.J. Taylor ${ }^{1}$, A. L. Herrick ${ }^{2}$, and T. Moore ${ }^{2}$ \\ 1 Wolfson Image Analysis Unit \\ Imaging Science and Biomedical Engineering \\ University of Manchester, Manchester M13 9PT, UK \\ pa@sv1.smb.man.ac.uk \\ http://www.wiau.man.ac.uk \\ 2 Rheumatic Diseases Centre \\ University of Manchester, Hope Hospital, Salford M6 8HD, UK
}

\begin{abstract}
Video capillaroscopy is a widely used technique to assess the condition of the blood capillaries in the nailfold of patients suffering from reduced peripheral circulation (Raynaud's phenomenon). This approach is complicated by the fact that gaps in the flow of blood can render capillaries incomplete in any single video frame. This paper describes a computer based system in which sequences of video frames are registered and combined to provide a composite image for analysis. We show that the images can be registered to an accuracy of approximately $2 \mu \mathrm{m}$, and that temporally variable features can be enhanced significantly by subtracting one standard deviation from the mean value for each pixel position in the video sequence.
\end{abstract}

\section{Introduction}

Among the symptoms produced by the connective tissue disease Scleroderma is a reduction in peripheral circulation exacerbated by exposure to cold (Raynaud's phenomenon). Extreme conditions can lead to amputation of fingers and toes and so improvement of the circulation in patients with Scleroderma is a major concern to clinicians. Trials aimed at this require an objective method of assessing the condition of the peripheral vasculature and quantifying changes caused by treatments under test.

One technique that has been explored is direct visualisation of the blood capillaries in the skin at the base of the finger nail (nailfold). Here the tiny vessels that link the arterial and venous system can be seen using an optical microscope (see figure 1). In previous work [1], a video camera has been coupled to the microscope, and the condition of the capillary network has been assessed by measuring certain key capillary loop dimensions from single video frames. Gaps in the flow of red blood cells through the capillary loops (the capillary wall itself is transparent) mean that not all of the capillary network is visible at any one instant. Thus it would be preferable to integrate the information from 
a sequence of successive video frames. Also, measuring the capillary dimensions by hand is tedious and therefore error prone.

This work forms part of an on going project to develop a computer based system for the analysis of nailfold capillary patterns in which the capillary dimensions will be measured automatically.

\section{Video Frame Registration}

The video information recorded by the clinicians is recorded in the form of a series of overlapping scenes. This is achieved by holding the finger as still as possible under the microscope for about 20 seconds or so, then moving the finger laterally on to the next scene, maintaining an overlap of one or more capillary loops. Two magnifications are used, $\times 200$ and $\times 600$. The low magnification images are used to give a general overview of the capillary structure, whilst the high magnification images are used for capillary dimension measurement. At the lower magnification, the region of the capillary network of interest is covered in three scenes. The aim of the system being developed is to register and combine the video frames from this material to form a single panoramic video mosaic showing the whole capillary network under study. Capillaries of interest identified in this mosaic can then be measured from their corresponding image in the $\times 600$ material.

For a sequence of video frames to be combined the small relative motions between finger and microscope during a sequence must be detected and corrected for, and in this application we require this process to be automatic. Accurate frame registration requires that the blood capillaries are separated from other elements in the image such as video noise or lens dust. Segmenting curvilinear structures such as blood vessels is a common problem in medical imaging [2]. The solution employed here is to apply a linear feature detector previously developed to detect line patterns in X-Ray mammograms [3]. The output of this process is a binary image containing a skeletal representation of the capillary structure where the capillaries are reduced to lines one pixel thick. From these binary images the transformation from one image to the next can be found. The extremely narrow depth of field inherent in optical microscopy means that changes in scale or perspective distortions are impossible. Thus the transformation from one frame to the next can be completely described by a combination of $2 \mathrm{D}$ translation and rotation. There are a variety of approaches to such an image registration problem [4]. Here the translation between two successive frames can be found from the skeletal representations using a Hough transform method. Each of the skeletal pixels from image A is matched with every skeletal pixel from image B. Each match represents a possible translation if rotation is known. If these matches are used to vote in a translation space, a peak emerges at the point representing the translation for which the largest number of pixels in the two images agree. If the two images are miss-aligned by a rotation as well as translation, the size of the peak in the translation image will be smaller than if there had been no rotation. Thus if the translation space is created for a number 
of possible rotations, the one which yields the highest peak corresponds to the correct, or at least best, rotation.

\section{$2.1 \quad$ Robustness}

The nature of the images in a video sequence can vary greatly from one patient to the next for two basic reasons. The first is the variation in image content caused by different capillary morphologies. In most healthy subjects the nailfold capillaries are in the form of long thin vertical loops arranged in roughly parallel horizontal rows (see figure 1), whereas the capillary loops of scleroderma patients tend to be enlarged and the regular row structure can become completely distorted (see figure 2). There can also be a complete absence of recognisable capillary loops in some places known as avascular regions.

The second source of image variability is caused by the condition of the skin. Viewing the nailfold capillaries with an optical microscope relies on the overlying skin being transparent, but one of the effects of scleroderma is to thicken the skin and this can reduce its transparency considerably.

Thus, designing a system that can successfully register and combine video frames corresponding to a single patient is no guarantee that it will work on any other patient, and so the system must be tested on as wide a set of patients as possible. The registration algorithms of the system being developed have been tested on the data of 25 patients so far. The 25 were chosen to include as wide a range of morphologies and image qualities as possible, and the results for two of the patients are shown in figures 1 and 2. The registration appears to be successful in all of the 25 cases even when, as in the case of overlapping scenes, the mutual information is relatively low.

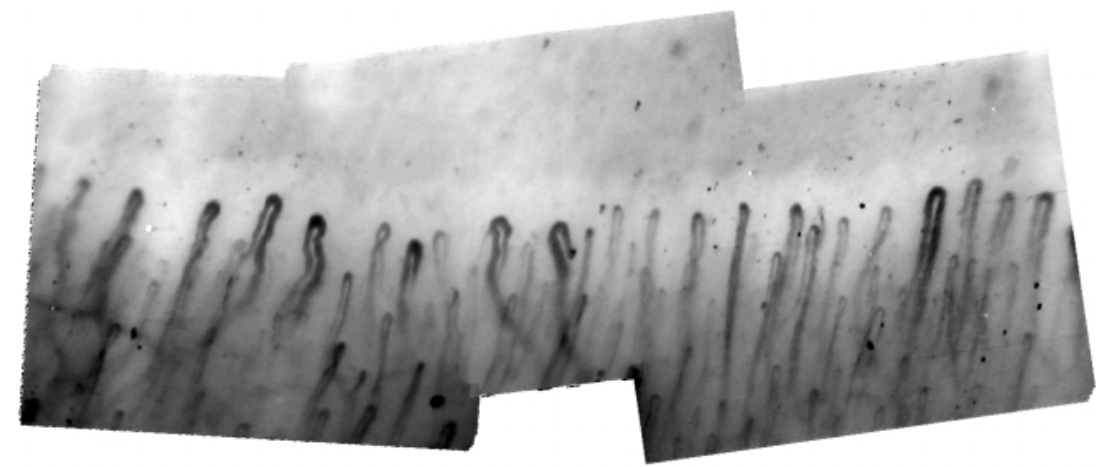

Fig. 1. Nailfold capillary structure at $\times 200$ magnification. This subject (patient $B$ in table 1) has scleroderma, but the capillary structure in this case exhibits the regular pattern of long thin loops typical of most healthy patients. The image shown is a composite of 48 video frames, 16 in each of the three overlapping scenes. 


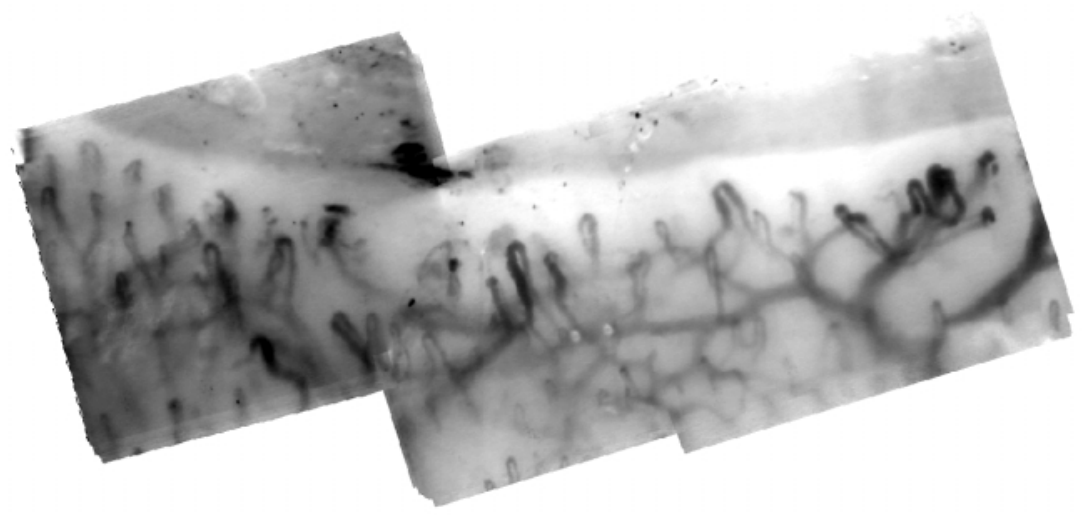

Fig. 2. Nailfold capillary structure at $\times 200$ magnification. This subject (patient $A$ in table 1) has diffuse scleroderma and exhibits distortion of the capillary loop pattern when compared with the more usual regular row structure visible in figure 1. The image shown is a composite of 48 video frames, 16 in each of the three overlapping scenes.

\subsection{Registration Accuracy}

To test the registration accuracy of the system a temporally stable feature is required in the scene which belongs to the tissue of the finger and hence reflects the motion of the capillary network through the sequence. In some of the patients (about 50\%), small dark specks about 6 pixels in diameter can be seen in the video frames. Examination of the moving video sequence reveals that some of these are specks of dust on the lens, but others belong to the finger's skin tissue, and are therefore ideal for testing registration accuracy.

Basically, a number of these dark specks are identified in each of the frames from a particular sequence and their positions noted. In each case the position recorded is the center of gravity of a small $(6 \times 6$ pixel $)$ region calculated as a geometric point rather than rounded to the nearest pixel position. The transformation vectors used to register the sequence of video frames are then applied to these points. If the registration was perfect, all of the points corresponding to the same image feature would be transformed to the same point. Instead, due to miss registrations, a cluster of points results and the spread in this cluster is a measure of registration accuracy.

Out of the 25 patient data sets examined so far, a set of four were chosen which exhibited point features suitable for testing registration accuracy and the results are shown in table 1 . For each patient there are one or more suitable scenes and each scene consists of either eight or sixteen video frames. Within each scene the number of usable image features, or points, will also vary and here the figures given are averaged over all the points used in the scene. 
Table 1. Estimates of image registration accuracy based on selected stable point like image features associated with skin tissue. The scene is simply the number of the scene used, $N_{F}$ is the number of video frames in that scene, and $N_{P}$ is the number of image points or features used for testing registrations accuracy. After image registration, clusters of points appear in th composite image corresponding to each of the selected features, and the standard deviation in $X$ and $Y$ from the mean position is taken to be the registration error. The $X$ and $Y$ errors (pixels) quoted are the average over all selected features in the scene, and the equivalent values for image combination without registration are shown for comparison.

\begin{tabular}{|c|c|c|c|c|c||c|c|}
\hline Patient & Scene & \multirow{2}{*}{$N_{F}$} & \multirow{2}{*}{$N_{P}$} & \multicolumn{2}{|c||}{ Registered } & \multicolumn{2}{c|}{ Unregistered } \\
\cline { 5 - 8 } & & & & X error & Y error & X error & Y error \\
\hline \hline \multirow{2}{*}{$\mathrm{A}$} & 2 & 16 & 3 & $0.64 \pm 0.07$ & $0.7 \pm 0.2$ & $7.13 \pm 0.08$ & $10.46 \pm 0.1$ \\
\cline { 2 - 8 } & 3 & 16 & 4 & $1.9 \pm 0.4$ & $1.9 \pm 0.3$ & $6.56 \pm 0.05$ & $17.80 \pm 0.08$ \\
\hline \hline \multirow{2}{*}{$\mathrm{B}$} & 1 & 16 & 13 & $0.91 \pm 0.08$ & $1.54 \pm 0.08$ & $3.77 \pm 0.09$ & $7.13 \pm 0.13$ \\
\cline { 2 - 8 } & 2 & 16 & 7 & $1.3 \pm 0.1$ & $1.22 \pm 0.09$ & $2.97 \pm 0.2$ & $6.43 \pm 0.07$ \\
\cline { 2 - 8 } & 3 & 16 & 6 & $1.1 \pm 0.1$ & $1.7 \pm 0.2$ & $5.12 \pm 0.1$ & $9.3 \pm 0.1$ \\
\hline \hline $\mathrm{C}$ & 3 & 8 & 6 & $1.0 \pm 0.1$ & $1.4 \pm 0.1$ & $1.45 \pm 0.1$ & $4.3 \pm 0.06$ \\
\hline \hline $\mathrm{D}$ & 2 & 8 & 3 & $1.4 \pm 0.2$ & $1.2 \pm 0.2$ & $3.57 \pm 0.2$ & $4.29 \pm 0.08$ \\
\hline
\end{tabular}

For each chosen feature a cluster of points (one for each video frame) will appear in the composite scene after registration. The average deviation from their geometric center in X and $\mathrm{Y}$ (horizontal and vertical)is quoted as the registration error. The reason that $\mathrm{X}$ and $\mathrm{Y}$ errors are determined separately rather than a simple scalar distance from the point cluster centroid is due to nature of the information in the nailfold images. For images in which the capillaries appear as long thin vertical loops there is less information from which to determine the $\mathrm{Y}$ displacement, and so a greater registration error in the $\mathrm{Y}$ axis may result. For example, the capillary loops of patient B in table 1 can be seen in figure 1 . Here, most of the capillaries are long thin loops and the results do indicate a greater registration error in the $\mathrm{Y}$ direction for scene 1 and 3.

The last two columns of table 1 show the registration errors derived if the points are not transformed according to the registration vectors. In other words these figures represent the spread we would expect in the position of the points belonging to a particular image feature if the video frames were simply combined without any attempt at registration. They show that there tends to be more vertical motion than horizontal. Here, vertical corresponds to the direction the finger is pointing and the larger displacements observed in this direction are simply a consequence of the way the finger is physically constrained during the microscopy session. This means that the greatest displacements between video frames will tend to be in the same direction as the capillary loops in subjects such as patient $\mathrm{B}$, and so how much of the extra registration error in the $\mathrm{Y}$ direction in this case is due to the nature of the image, and how much is due 
simply to the fact that most of the movement was in this direction anyway, is difficult to assess.

At the moment there are only a small number of patients exhibiting suitable features to test registration accuracy and larger numbers will be utilised to obtain a more definitive assessment of the system's performance. From the data analysed so far we can say that the registration errors are less than two pixels in either $\mathrm{X}$ or $\mathrm{Y}$. Since we expect the registration accuracy to be dependent on the capillary structure it is less meaningful to quote averages over a number of patients than to focus on the worst cases. In the current manual system the capillary dimensions are measured from single video frames and so the limiting factor is the image resolution i.e. any dimension measured is known to \pm 1 pixel (corresponding to approximately $2 \mu \mathrm{m}$ ). However, the noise levels in a single frame is considerably higher than in a composite scene and this will reduce the accuracy to which a feature's dimensions can be measured. Also of course, temporal variability may result in a feature being completely absent in a particular scene. The registration accuracy exhibited so far is therefore acceptable.

\subsection{Combining Images}

Once a sequence of video frames has been registered with respect to the capillary network, the information from the set of images has to be combined in such a way as to make maximum use of the available information. The most obvious approach is to take the mean of the image set. This is the most desirable way of enhancing stable image features, as the random noise present in the individual images is averaged out. However, if we consider the case of a capillary in which the column of red blood cells contains visible gaps, at any given point on the capillary the intensity of the image will vary with time as the 'lumps' of blood flow through. If an average of the sequence is taken, this variation between light (background) and dark (blood cells) will result in an average value in between and so the contrast between capillary and background will be lower than it was in a single frame. Thus, the complete capillary becomes visible, but contrast is lost. Another commonly used approach is to select the minimum value for each pixel from the sequence. This improves signal contrast, but involves no noise averaging, so it is not obvious that the visibility of the results will be any better than taking the mean. We propose a third alternative, which is to subtract some multiple of the standard deviation from the mean. The idea is to estimate a value representative of the minimum over the sequence but less susceptible to noise.

If the contrast between a capillary and the surrounding tissue in a composite scene can be quantified, then the relative merits of the image combination techniques described above can be assessed. If we consider the difference between the intensity of the capillary and the surrounding tissue as the signal, then the contrast can be quantified as the signal to noise ratio. To define the signal to noise ratio mathematically we must make assumptions about the average grey level intensities of blood and background tissue, and what the noise will be on these values. These can be estimated from the nail-fold images encountered in 
this study. The other important variables to consider are the fraction of time $F_{S}$ that a particular pixel's grey level intensity corresponds to blood, and the number of images in the sequence $N . F_{S}$ can also be thought of as a measure of the capillary's temporal variability - a low $F_{S}$ would correspond to a capillary in which the gaps in the flow of blood were large compared to the length of the column of red blood cells, and visa versa.

There is insufficient space in this paper to go through the resulting derivations [5], however, figure 3 shows the predicted relationship between signal to noise ratio and $F_{S}$ for the mean image, minimum projection, and $\mu-n \sigma$, when the number of frames in the sequence $N$ is 100 . The best results are obtained from $\mu-n \sigma$, but the optimum value of $n$ is a function of $F_{S}$.

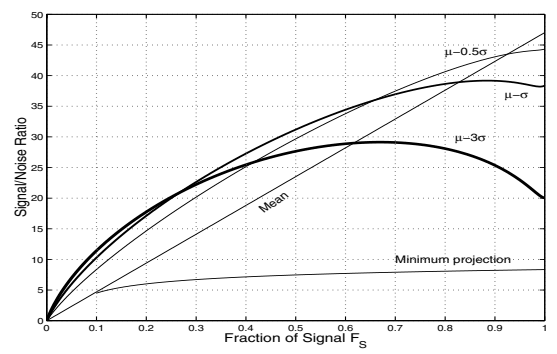

Fig. 3. Signal to noise ratio as a function of feature variability $F_{S}$.

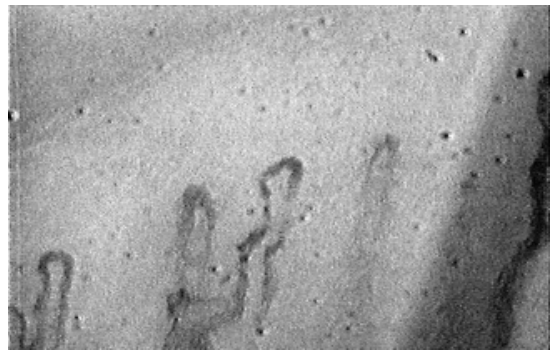

Fig. 4. Single video frame of nailfold capillaries at $\times 600$ magnification.

\section{Applying Image Combination Techniques to Data}

The discussion in section 2.3 is partly illustrated in figures 4 to 6 . Figure 4 shows a single frame from a sequence of 20 , the central capillary in this scene displays large gaps in the flow of blood and so the capillaries appearance was variable with time.

Figure 5 shows the result of taking the mean of the image sequence. The central capillary loop now appears complete and the noise in the scene has been reduced. The capillary loops either side of the central loop still appear relatively indistinct - this is because they are slightly deeper in the skin than the others. With the reduction in noise, the texture of the skin obscuring them is just becoming apparent. Figure 6 shows the result of subtracting one standard deviation from the mean image. The contrast of the central capillary has been enhanced but there has been a slight increase in the noise levels of the surrounding temporally stable background. 


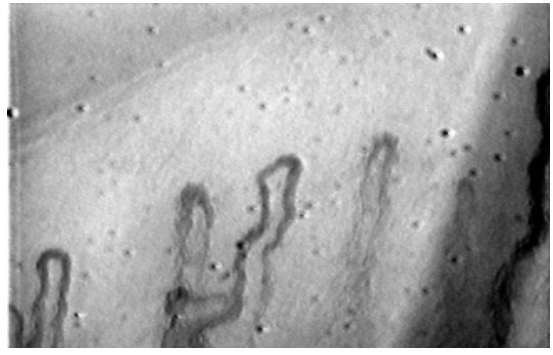

Fig. 5. The mean pixel values of the registered video frame sequence.

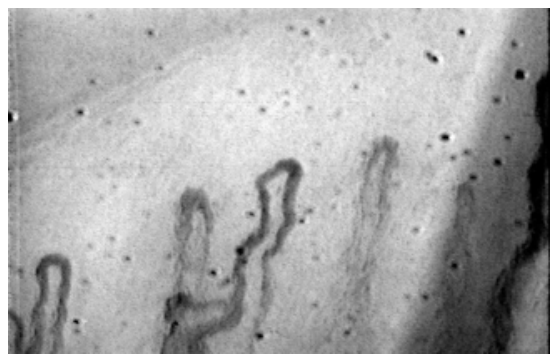

Fig. 6. The mean image minus one standard deviation.

\section{Conclusions}

So far this system has only been tested on 25 patient data sets, but the indications are that the video frame registration is robust and acceptably accurate.

The signal to noise ratio estimates in figure 3 show that for the noise and contrast levels found in nailfold images, the best signal to noise ratio is achieved by using $\mu-n \sigma$, where the value of $n$ is a function of the degree of temporal variability $F_{S}$. If the contrast between blood cells and surrounding tissue and the noise level $\sigma$ is known and can be assumed to be constant, then the value of $F_{S}$ can be derived at each pixel position from the equations on which figure 3 was based. However, the blood/background contrast varies from one patient to the next. The extent of this variation and whether this will render evaluation of $F_{S}$ impracticable requires further study. However, figure 3 suggests that subtracting between a half and one standard deviation gives an improved signal to noise ratio over the mean for most values of $F_{S}$. Also the set of images in section 3 show that using $\mu-\sigma$ improves the contrast of the variable capillary without noticeable degradation of stable ones. Thus, in the absence of a reliable estimate of $F_{S}$, $\mu-\sigma$ would seem to be the best option for image combination of the nailfold images encountered in this study.

These results are also of general application to problems requiring temporal or spatially varying data to be compounded.

\section{References}

1. M. Bukhari, A.L. Herrick, T. Moore, J. Manning, and M. I. V. Jayson. Increased Nailfold Capillary Dimensions in Primary Raynaud's Phenomenon and Systemic Sclerosis. British Journal of Rheumatology, 35:1127-1131, 1996.

2. R. Kutka and S. Stier. Extraction of Line Properties Based on Direction Fields. IEEE Trans. Med. Imag, 15(1):51-58, February 1996.

3. R. Zwiggelar, T. Parr, and C. Taylor. Finding Oriented Line Patterns in Digital Mammographic Images. In Proc. of 7th BMVC Edinburgh, 1996.

4. L. G. Brown. A Survey of Image Registration Techniques. ACM Computing Surveys, 24(4):321-376, December 1992.

5. P. D. Allen, C. J. Taylor, A.L. Herrick, and T. Moore. Enhancement of Temporally Variable Features in Nailfold Capillary Patterns. In British Machine Vision Conference, volume 2, pages 535-544, 1998. 